\title{
Editorial
}

\section{The Impact of Climate Change on the Emerging and Re-emerging Infectious Diseases: Global Perspective}

Climate change is one of the most complex challenges of the century, affecting all countries. Globalization and climate change have caused an unprecedented worldwide impact on emerging and re-emerging diseases including zoonoses in the recent years. ${ }^{1}$ Emerging infectious diseases refer to diseases caused by newly identified and previously unknown infectious agents that cause public health problems either locally or internationally, while reemerging infectious diseases are caused by infectious agents that have been known for some time, had fallen to such low levels that they were no longer considered public health problems and are now showing upward trends in incidence or prevalence worldwide. Microbes and vectors swim in the evolutionary stream, and they swim faster than we do. Global warming and climate change have taken diseases like malaria, dengue and other vector-borne diseases to new dimensions. Climatic factors, particularly temperature and rainfall, affect the ability of malarial parasites, viral propagation and potential mosquito vectors to coexist long enough to maintain and increase the rate of transmission. ${ }^{2}$

The decreased prevalence of infectious diseases in western countries in 20th century was because of urban sanitation, improved housing, personal hygiene, antisepsis and vaccination. Introduction of antibiotics further suppressed morbidity and mortality. Since last quarter of 20th century, there has been resurgence of infectious diseases and the world remains in transition by the fact that certain infectious diseases (AIDS, avian influenza, Ebola, Marburg, cholera, Rift Valley fever, typhoid, tuberculosis, leptospirosis, malaria, chikungunya, dengue, Japanese encephalitis) have emerged or reemerged while situations with others (guinea worm, smallpox, yaws, poliomyelitis, measles, leprosy and neonatal tetanus) have declined significantly. 3,4
Zoonoses-transmission of infectious agent from animals (wild and domestic) to humans constituted more than two-thirds of emerging infections. Contact among wild animals, domestic animals and people is another driving force involving the interaction between naïve population groups that induces the emergence of new diseases. Deforestation forces animals into closer human contact - increased possibility for agents to breach species barrier between animals and humans responsible for diseases like Lassa fever, Yellow fever and malaria while global warming is facilitating spread of vector-borne diseases like malaria, dengue, leishmaniasis and filariasis. ${ }^{5}$ Uncontrolled urbanization and population displacement have given rise to growth of densely populated cities with substandard housing, unsafe water, poor sanitation, overcrowding, indoor air pollution with consequent incidence of diarrhea, intestinal parasitic diseases, Lyme disease and ARI. ${ }^{6}$

The current state of attention believes the fact that emerging infections are not a recent phenomenon but have always played a major role throughout human history. ${ }^{3}$ Recognizing the complexity of the diverse sociocultural processes involved in the reemergence/emergence of infectious disease, many researchers in biology, medicine, and public health are calling for input from the social and behavioral sciences. With its integrative approach to complex biocultural issues, anthropology is well positioned to make significant theoretical and practical contributions.

Climate change has been responsible for at least one emerging or re-emerging disease in many countries and the number of such countries is gradually increasing. Hepatitis $\mathrm{C}$ virus, although first identified in 1989, in mid-1990s estimated global prevalence was 3\%. Hepatitis B, identified several 
decades earlier, has shown a remarkable upward trend in all countries, in particular South-East Asian high-risk population. The first case of HIV/AIDS in Bangladesh was detected in 1989, and since then 1,745 HIV positive cases have been identified in whom 619 developed AIDS till December 2009. Out of the total AIDS cases, 204 deaths have been recorded till December 2008. Severe acute respiratory syndrome (SARS), the first emerging infectious disease of the $21^{\text {st }}$ century in one country is an alarming threat to all air travelers with a tremendous negative economic impact on trade, travel and tourism. ${ }^{7}$ Nipah virus infection has been becoming endemic in Bangladesh as cases are being continuously detected since 2001. Avian influenza (H5N1) has emerged since November 2003 in birds and affected 60 countries across Asia, Europe, Middle-East and Africa, and more than 220 million birds were killed by the virus or culled to prevent further spread. Novel swine origin influenza A (H1N1) leading to swine flu causes respiratory disease in pigs implicated with high level of illness, low death rates. It is noteworthy that pigs can get infected by human, avian and swine influenza viruses. In late 2009 and early 2010, there was a great panic due to global pandemic of swine flu. Although a few positive cases of swine flu were detected, there were only two recorded deaths in Bangladesh. $^{7}$

Re-emerging infectious diseases include diphtheria epidemic in Eastern Europe in early 1990s, cholera was associated with $100 \%$ increase worldwide in 1998 (new strain Eltor, 0139), human plague was reported from India in 1994 after 15-30 years absence. The outbreak of dengue has occurred over past 40 years with 20 -fold increase to nearly 0.5 million from 1990 to 1998 . The medical communities of Bangladesh were fairly unfamiliar about the presence of dengue in Bangladesh before 2000. Since its outbreak started in summer of 2000 , every year some cases are being reported. Chikungunya fever is also a re-emerging condition in previously unaffected areas with possibly changing epidemiology and severity of the disease. Chikungunya tends to cluster geographically and overlap with dengue because they share some common features. ${ }^{8}$
The role of climate as well as environmental changes on the growing burden of emerging and reemerging disease call for a new approach to prevent these new threats. The risk factors and response options need to be appropriate for the nature of vulnerabilities that might affect the demographic transitions due to climate change. Health, nutrition and population experts must address these areas of public health issues related to climate change with the required responses. Member countries have consequently given the World Organization for animal health a mandate to address the issue by using its scientific capabilities and networks at global, regional and sub-regional levels with the aim of preventing or reducing the effects of climate change on animal diseases which are transmissible to humans. ${ }^{4}$

In order to offer a multidisciplinary perspective to mitigate the problem, infectious disease specialists, epidemiologists, geneticists, microbiologists, and population biologists need to participate to address questions about the definition, the identification, the factors responsible for, and multidisciplinary approaches to emerging and reemerging infections. There is a need for surveillance at national, regional and global levels by epidemiological, laboratorybased, ecological, and anthropological approach to adapt early control measures.

The role of public health professionals is to establish surveillance for unusual diseases, drug resistant agents, to assure laboratory capacity to investigate new agents (eg, high-throughput laboratories), to develop plans for handling outbreaks of unknown agents and to inform physicians about rational antimicrobial use. Finally, high level national and international political commitment is crucial for rapid containment of this global issue.

\section{Iftikhar Ahmed}

Professor

Department of Microbiology

Enam Medical College, Savar, Dhaka 


\section{References}

1. Gummow B. Challenges posed by new and re-emerging infectious diseases in livestock production, wildlife and humans. Livestock Science 2010; 130: 41-46.

2. Kumar A, Valecha N, Jain T, Dash AP. Burden of malaria in India: retrospective and prospective view. Am J Trop Med Hyg 2007; 77: 69-78.

3. Stephens DS, Moxon ER, Adams J et al. Emerging and reemerging infectious diseases: a multidisciplinary perspective. American Journal of the Medical Sciences 1998; 315: 64-75.

4. Armelagos GJ. Disease, Darwin and medicine in the third epidemiological transition. Evol. Anthropol. 1997; 5: 212-220.
5. Ramaiah KD, Das PK, Michael E, Guyatt HL. The economic burden of lymphatic filariasis in India. Parasitol Today 2000; 16: 251-253.

6. Joshi A, Narain JP, Prasittisuk C, Bhatia R, Hashim G, Jorge A, Banjara M. Kroeger A. Can visceral leishmaniasis be eliminated from Asia? J Vector-borne Dis 2008; 45: 105-111.

7. Lederberg J. Infectious disease as an evolutionary paradigm. Emerg. Infect. Dis. 1997; 3: 417-423.

8. Warter L, Lee CY, Thiagarajan R et al. Chikungunya virus envelope-specific human monoclonal antibodies with broad neutralization potency. The Journal of Immunology 2011; 186: 3258. 